\title{
Discussion on Calculation Method of Positioning Error
}

\author{
Shuping Lv \\ Dalian Vocational \& Technical College, Dalian, China \\ 964511001@qq.com
}

Keywords: Process reference; Positioning reference; Process size; Positioning error

\begin{abstract}
During the machining process, in order to ensure the machining accuracy of the workpiece, the workpiece occupy a certain position relative to the machine tool and the tool, and it also can be clamped quickly and reliably, this process equipment that accepts processing or inspection is called the machine tool fixture. The analysis and calculation of positioning error occupies an important position in the fixture design. The size of the positioning error is an important basis for determining the positioning scheme. The calculation of positioning error is a difficult point, and sometimes it cannot be started. Therefore, this article will give a sets of concrete steps make its calculations quick and accurate and verified by examples.
\end{abstract}

\section{Positioning Error and Its Cause}

In the fixture, the task of the workpiece positioning is to make the same batch of workpiece occupy the correct machining position. In fact, due to the manufacturing error of the positioning reference and positioning elements, the position of the same batch of workpieces in the fixture may not be the same. This change in position will result in an error in the machining dimensions. That is, the maximum amount of positional deviation in the direction of process size in the process reference, which is referred to as positioning error, is represented by $\Delta \mathrm{D}$.

Whether the accuracy of the workpiece can be guaranteed depends on the correct mutual position between the tool and the workpiece. There are several error factors that affect this correct positional relationship, as follows:

(1) Positioning error $-\Delta_{D}$.

(2) Installation error and adjustment error- $-\Delta_{T-A}$.

(3) Process error $-\Delta_{G}$.

In order to ensure processing requirements, the above three errors should be less than or equal to the tolerance of the workpiece $\delta_{K}$, that is $\Delta_{D}+\Delta_{T-A}+\Delta_{G} \leq \delta_{K}$.

The above three errors should be less than or equal to the tolerance of the workpiece $\delta_{K}$, that is $\Delta_{D}+\Delta_{T-A}+\Delta_{G} \leq \delta_{K}$. In the analysis of the positioning scheme, it can be assumed that the above three errors hold $1 / 3$ of the workpiece tolerance respectively. As long as process size difference of workpiece caused by the position change in the fixture, it does not exceed the allowable range specified in this procedure, and then the position where the workpiece has been determined in the fixture is considered to be correct.

\section{The Composition and Calculation Formula of Positioning Error}

When a batch of workpieces is installed by fixture and processed by an adjustment method, the maximum variation range of the processing size due to the positioning is called a positioning error, is represented by $\Delta_{D}$. The positioning error consists of two parts, the reference misalignment error and the reference displacement error. 


\subsection{The Reference Misalignment Error $\Delta_{B}$.}

The maximum positional deviation in the direction of the process size of the process reference relative to the positioning reference caused by misalignment between the positioning reference and the process reference is called the reference non-coincidence error, is represented by $\Delta_{B}$.

If the positioning size is in the same direction as the process size, the size of the reference misalignment error is equal to the dimensional tolerance between the positioning reference and the process reference (or the tolerance of the positioning size).

If the positioning size does not match the process size, the size of the reference misalignment error is equal to the projection of the positioning size tolerance in the direction of the processing size (ie, process size).

\subsection{The Reference Displacement Error $\Delta_{Y}$.}

When the workpiece is positioned in the fixture, due to the influence of the manufacturing tolerances of the positioning pair (the positioning surface of the workpiece and the working surface of the positioning element) and the minimum matching clearance, the positioning reference is displaced in the direction of the processing size, resulting in inconsistent positions for each of workpieces. The maximum positional deviation of the workpiece positioning reference in the direction of the machining dimension is referred to as the reference displacement error for this batch of workpieces, is represented by $\Delta_{Y}$.

The positioning error is the combined result of the reference displacement error and the reference misalignment error. It can be represented as:

$$
\Delta_{D}=\left|\Delta_{Y} \pm \Delta_{B}\right|
$$

The positive and negative signs of positioning error can be judged separately by the process reference is on the positioning base surface or not.

(1) Process reference is on the positioning base surface, When the reference displacement and the reference misalignment respectively cause the processing size to be changed in the same direction (that is, Increase or decrease simultaneously), a positive sign is taken; and when the reference displacement and the reference misalignment respectively cause the processing size changing in the opposite direction for each other, the negative sign should be taken.

(2) Process reference is not on the positioning base surface. At this time, the reference misalignment error takes the positive sign, that is, the positioning error is the sum of the reference displacement error and the reference misalignment error, so $\Delta_{D}=\Delta_{Y}+\Delta_{B}$.

It is necessary to emphasize here that the positioning error mainly occurs when a batch of workpieces is processed by the adjustment method. If machining is done piece by piece, there is no positioning error.

When calculating the positioning error, the process size and the corresponding process reference should be clearly defined firstly, and based on the positioning method, to determine whether the positioning reference and the process reference are coincident. If they are coincident, then $\Delta_{B}=0$. If not, firstly to find the connection size between the process reference and positioning reference (ie, the positioning size), the projection in the direction of the process size is the reference misalignment error. Secondly, to calculate the reference displacement error based on the positioning method, and finally, the positioning error is calculated by combined these two error.

\section{Calculate Positioning Error Steps}

For the analysis and calculation of positioning error, to summarize the calculation steps as follows:

(1) Firstly, to define the processing surface of this process;

(2) Requested guaranteed fabrication dimensions (process dimensions) and tolerances; 
(3) The process reference of this process size;

(4) The positioning reference during processing;

(5) To determine if there is a positioning misalignment errors;

(6) The reference misalignment positioning error is equal to the dimensional tolerance between the process reference and the positioning reference (that is, the positioning dimension is in the same direction as the process dimension, otherwise it is the projection on the processing dimension)

(7) The reference displacement error calculation for common positioning methods;

(a) The workpieces is positioned by plane $\Delta_{Y}=0$.

(b) The workpieces is positioned by plane cylindrical hole.

(i) The workpiece is positioned with a cylindrical hole on the interference fit mandrel $\Delta_{Y}=0$.

(ii) The workpiece is positioned with a cylindrical hole on the interstitial cylindrical spindle. The hole touch with mandrel on the fixed side:

$$
\Delta_{Y}=\frac{1}{2}\left(D_{\max }-D_{\min }\right)=\frac{1}{2} \delta_{D}+\frac{1}{2} \delta_{d}
$$

The hole touch with cylindrical spindle on any side:

$$
\Delta_{Y}=D_{\max }-d_{\min }+X_{\min }=\delta_{D}+\delta_{d}+X_{\min }
$$

(c) The workpiece is positioned by outer circle on the V-shape block:

$$
\Delta_{Y}=\frac{\delta_{d}}{2 \sin \frac{\alpha}{2}}
$$

(8) Synthesis of positioning errors:

$$
\Delta_{D}=\Delta_{Y} \pm \Delta_{B}
$$

(9) Synthesis method for positioning errors:

(a) When both $\Delta_{Y}$ and $\Delta_{B}$ are zero, $\Delta_{D}$ is also zero.

(b) When both $\Delta_{Y}$ and $\Delta_{B}$ are not zero, the positive and negative signs of positioning error can be judged separately on the positioning base surface according to the process reference.

(i) Process reference is on the positioning base surface, When the reference displacement and the reference misalignment respectively cause the processing size to be changed in the same direction (that is, Increase or decrease simultaneously), a positive sign is taken; and when the reference displacement and the reference misalignment respectively cause the processing size changing in the opposite direction for each other, the negative sign should be taken.

(ii) Process reference is not on the positioning base surface. At this time, the reference misalignment error takes the positive sign, that is, the positioning error is the sum of the reference displacement error and the reference misalignment error, so $\Delta_{D}=\Delta_{Y}+\Delta_{B}$.

It is necessary to emphasize here that the positioning error mainly occurs when a batch of workpieces is processed by the adjustment method. If machining is done piece by piece, there is no positioning error.

\section{The Example of Positioning Error Calculation}

Example 1: Slot on the stepped shaft, to ensure the dimension of $\mathrm{H}$ and L. Blank dimension is $D=\phi 160_{-0.14}^{0} \mathrm{~mm}, d=\phi 40_{-0.1}^{0} \mathrm{~mm}, D$ has a coaxial error of $0.04 \mathrm{~mm}$ to $d$. Positioning plan as below drawing. Try to calculate positioning error of size $H$ and $L$ ( $V$-block angle is $90^{\circ}$ ). 

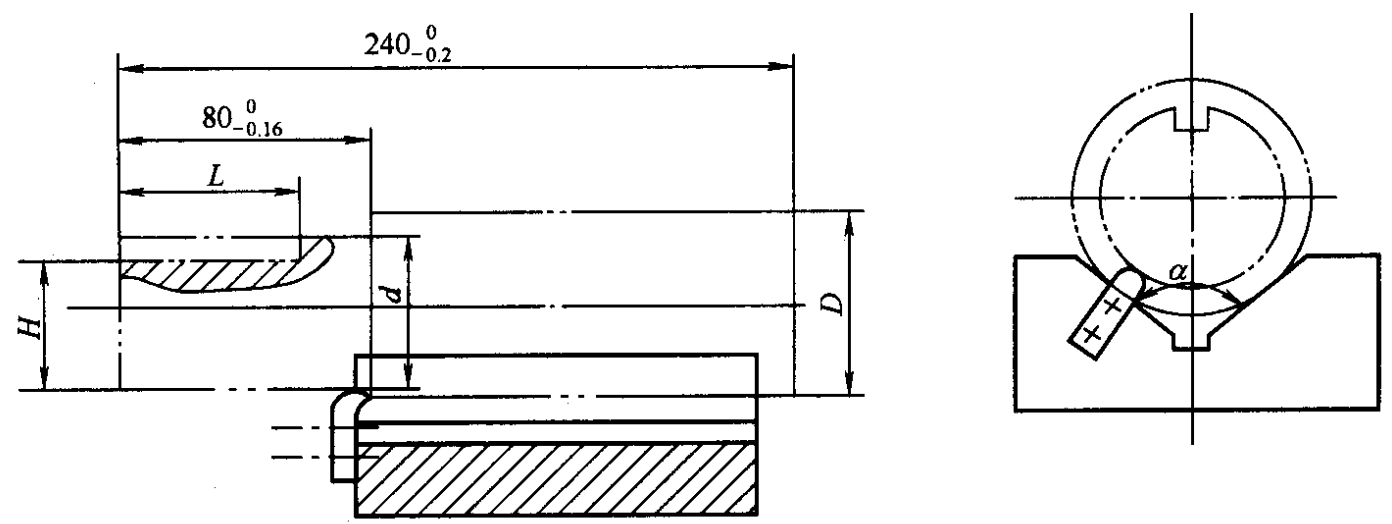

Fig.1 The drawing of slot on the stepped shaft

Based on the above summarized calculation steps, as follows:

(1) The processed surface is a key slot in this process.

(2) The required fabrication dimensions (process dimensions) are $L$ and $H$.

(3) The process references are respectively the left surface and the bottom bus of the outer circle d.

(4) Positioning references during machining are respectively the $D$ left surface and the center line of $D$.

(5) Determine the reference misalignment error. The reference misalignment error of $L$ is $\Delta_{B}=0.16 \mathrm{~mm}$; the reference misalignment error of $H$ is $\Delta_{B}=0.05+0.04=0.09 \mathrm{~mm}$.

(6) The reference displacement error calculation:

To size $L$, the workpieces is positioned by $\Delta_{Y}=0$ plane.

To size $H$, the workpieces is positioned by V-shape $\Delta_{Y}=\delta_{d} /\left(2 \sin \frac{\alpha}{2}\right)$ block:

(7) According to the summarized method of positioning error, as follows.

The positioning error for $L$ :

$$
\Delta_{B}=0.16 \mathrm{~mm}, \Delta_{Y}=0 \mathrm{~mm}, \Delta_{D}=0.16+0=0.16 \mathrm{~mm}
$$

The positioning error for $\mathrm{H}$ :

$$
\Delta_{B}=0.05+0.04=0.09 \mathrm{~mm}, \Delta_{Y}=0.707 \times 0.14=0.099 \mathrm{~mm}, \Delta_{D}=0.09+0.099=0.189 \mathrm{~mm}
$$

Example 2: As below drawing, stepped shaft positioning on V-shaped frame (double V-shaped frame), drilling $D$ and milling half moon key slot. As known, $d_{1}=\phi 35_{-0.017}^{0} \mathrm{~mm}, d_{2}=\phi 35_{-0.050}^{-0.025} \mathrm{~mm}$, $L_{1}=80 \mathrm{~mm}, L_{2}=30 \mathrm{~mm}, L_{3}=120 \mathrm{~mm}, L_{3}=120 \mathrm{~mm}, \alpha=90^{\circ}$. If don't consider the manufacturing error of the $\mathrm{V}$-shaped bracket, try to calculate the positioning error of size $A_{1}$ and $A_{2}$. (When $\alpha=90^{\circ}$, the $\mathrm{V}$-block positioning error value is $0.707 \delta_{D}$ )
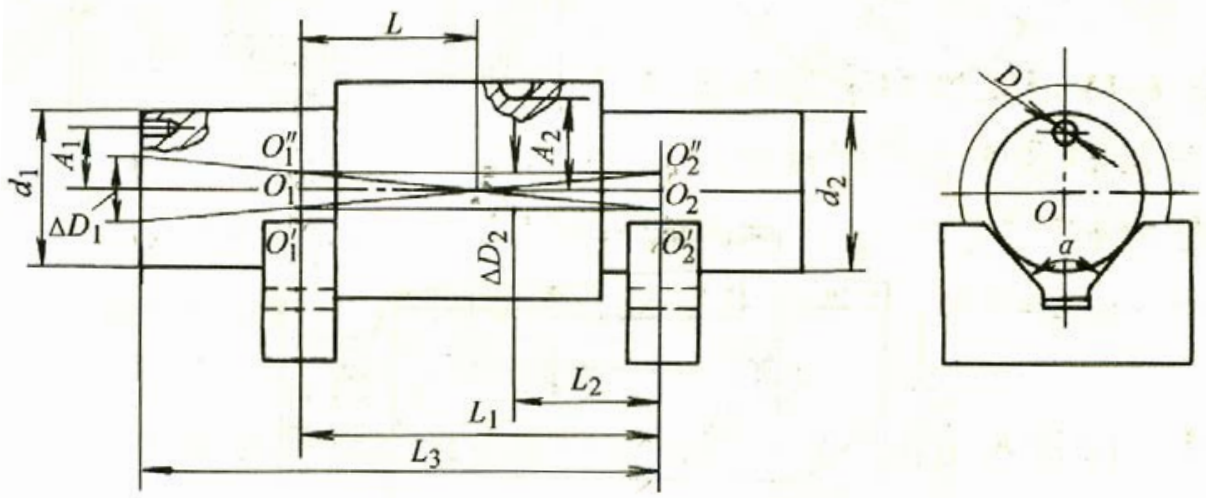

Fig.2 The drawing of stepped shaft positioning on V-shaped frame 
Based on the above summarized calculation steps, as follows:

(1) The processed surface is small hole and semi-circular key slot in this process.

(2) The required fabrication dimensions (process dimensions) are $A_{1}$ and $A_{2}$.

(3) The process references of this process size.

(4) Positioning references during machining is the shaft center line.

(5) Determine the reference misalignment positioning error, reference coincidence $\Delta_{B}=0$.

(6) The workpiece is positioned by outer circle on the V-shape block, and reference displacement error is:

$$
\begin{array}{r}
\Delta_{Y}=\frac{\delta_{d}}{2 \sin \frac{\alpha}{2}} \\
0_{1}^{r} \mathrm{O}_{1}^{\mathrm{m}}=0.707 \delta \mathrm{d}_{1}=0.707 \times 0.017=0.012 \mathrm{~mm} \\
\mathrm{O}_{2}^{r} \mathrm{O}_{2}^{\mathrm{m}}=0.707 \delta \mathrm{d}_{2}=0.707 \times 0.025=0.018 \mathrm{~mm}
\end{array}
$$

To Size $A_{1}$, the max variation of processing reference is $\Delta_{D 1}$. To Size $A_{2}$, the max variation of processing reference is $\Delta_{D 2}$.

$$
\begin{array}{r}
\mathrm{O}_{1}^{\prime} \mathrm{O}_{1}^{\mathrm{m}}: \mathrm{O}_{2}^{\prime} \mathrm{O}_{2}^{\mathrm{m}}=\mathrm{L}:\left(\mathrm{L}_{1}-\mathrm{L}\right) \text { so: } \mathrm{L}=32 \mathrm{~mm} \\
\Delta \mathrm{D}_{1} / \mathrm{O}_{1}^{\prime} \mathrm{O}_{1}^{\mathrm{m}}=\left(\mathrm{L}_{3}-\mathrm{L}_{1}+\mathrm{L}\right) / \mathrm{L}
\end{array}
$$

So

$$
\begin{gathered}
\Delta \mathrm{D}_{1}=0.027 \mathrm{~mm} \\
{\left[\left(\mathrm{O}_{2}^{\prime} \mathrm{O}_{2}^{\mathrm{m}}-\mathrm{O}_{1}^{\prime} \mathrm{O}_{1}^{\mathrm{m}}\right) / 2\right] / \mathrm{L}_{1}=\mathrm{X} /\left(\mathrm{L}_{1}-\mathrm{L}_{2}\right)} \\
\Delta \mathrm{D}_{2}=\mathrm{O}_{1}^{r} \mathrm{O}_{1}^{\mathrm{m}}+2 \mathrm{X}=0.012+\frac{[(0.018-0.012) \times(80-30)]}{80}=0.016 \mathrm{~mm}
\end{gathered}
$$

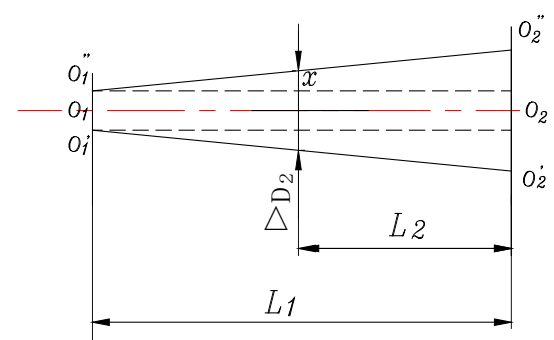

Fig.3 The drawing of the max variation of processing reference

\section{Conclusion}

The size of positioning error in the fixture design is an important basis to determine the positioning scheme. The calculation of positioning error is a difficult point, and sometimes it is impossible to start. Through the above analysis, it can be seen that different positioning methods have different positioning error values. A set of specific steps makes it quick and accurate to calculate, calculate the positioning error value of different positioning schemes, do not need to derive complex geometric relations, which greatly simplifies the solution process.

\section{References}

[1] Zhao Hongli. Mechanical Manufacturing Technology and Equipment. Beijing: People's Posts and Telecommunications Press, 2014.

[2] Wang Yijun. Manufacturing Technology Foundation. Beijing: Qinghua University Press, 2015. 
[3] Liu Shouyong. Mechanical Manufacturing Technology and Machine Tool Fixture. Beijing: Mechanical Industry Press, 2014.

[4] Zhu Shuping. Mechanical Manufacturing Technology and Equipment. Beijing: Mechanical Industry Press, 2010.

[5] Xiao Jide. Machine tool fixture design. Beijing: Mechanical Industry Press, 2016.

[6] Lan Jianshe. Mechanical Manufacturing Technology and Fixtures. Beijing: Mechanical Industry Press, 2015. 